Strain induced enhanced ferromagnetic behavior in inhomogeneous low doped La $0.95 \mathrm{Sr}_{0.05} \mathrm{MnO}$ $3+\delta$

S. Das', J. S. Amaral', K. De', M. Willinger, J. N. Gonçalves, A. Roy, P. Dhak, S. Giri, S. Majumder, C. J. R. Silva, M. J. M. Gomes, P. K. Mahapatra, and V. S. Amaral

Citation: Appl. Phys. Lett. 102, 112408 (2013); doi: 10.1063/1.4793657

View online: http://dx.doi.org/10.1063/1.4793657

View Table of Contents: http://aip.scitation.org/toc/apl/102/11

Published by the American Institute of Physics

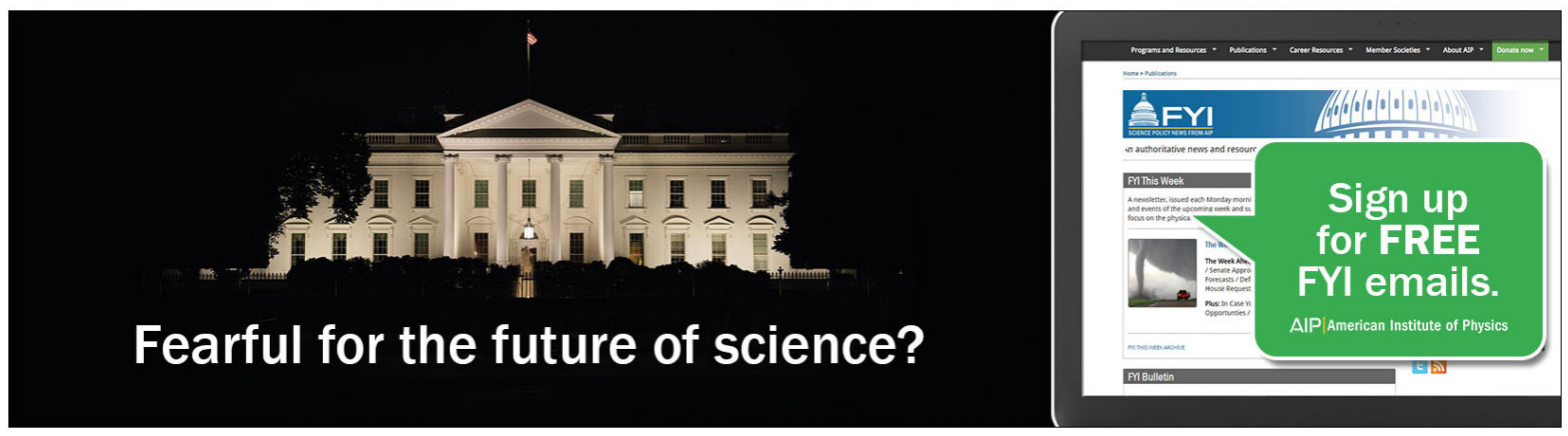




\title{
Strain induced enhanced ferromagnetic behavior in inhomogeneous low doped $\mathrm{La}_{0.95} \mathrm{Sr}_{0.05} \mathrm{MnO}_{3+\delta}$
}

\author{
S. Das, ${ }^{1,2, a)}$ J. S. Amaral, ${ }^{1,2,3, b)}$ K. De ${ }^{4,5, c)}$ M. Willinger, ${ }^{2}$ J. N. Gonçalves, ${ }^{1,2}$ A. Roy, ${ }^{6}$ \\ P. Dhak, ${ }^{7}$ S. Giri, ${ }^{8}$ S. Majumder, ${ }^{8}$ C. J. R. Silva,${ }^{9}$ M. J. M. Gomes, ${ }^{9}$ \\ P. K. Mahapatra, ${ }^{10}$ and V. S. Amaral ${ }^{1,2}$ \\ ${ }^{1}$ Departamento de Física, Universidade de Aveiro, Aveiro 3810 193, Portugal \\ ${ }^{2}$ CICECO, Universidade de Aveiro, Aveiro 3810 193, Portugal \\ ${ }^{3}$ IFIMUP IN and Department of Physics and Astronomy, University of Porto, Porto 4169007 , Portugal \\ ${ }^{4}$ Department of Physics and Technophysics, Vidyasagar University, Midnapore 721102, India \\ ${ }^{5}$ NITMAS, Jhinga, D. H. Road, 24 Pgs (S) 743 368, India \\ ${ }^{6}$ Dinhata Mission Girls' High School, Coochbehar 736135, India \\ ${ }^{7}$ Department of Chemistry, Indian Institute of Technology, Kharagpur 721302, India \\ ${ }^{8}$ Department of Solid State Physics, Indian Association for the Cultivation of Science, \\ Jadavpur, Kolkata 700032, India \\ ${ }^{9}$ Physics Centre and Centre of Chemistry, University of Minho, Braga 4710 057, Portugal \\ ${ }^{10}$ West Bengal University of Technology, Bidhannagar, Kolkata 700072, India
}

(Received 7 October 2012; accepted 14 February 2013; published online 19 March 2013)

\begin{abstract}
We report an unusual high-temperature ferromagnetic transition in bulk single-phase nanocrystalline $\mathrm{La}_{0.95} \mathrm{Sr}_{0.05} \mathrm{MnO}_{3+\delta}$, achieved through localized strain and inhomogeneous Sr-doping. Magnetization measurements show a well defined transition at $290 \mathrm{~K}$ and a broad one at $\sim 150 \mathrm{~K}$. HRTEM imaging reveals the strain on the highly crystalline nanometer sized grains and Sr-doping gradients, while oxygen homogeneity at the grain interfaces is confirmed by EELS-spectra. The magnetic behavior, far from the expected bulk phase diagram, shows how local doping and strain can strongly tune the macroscopic properties of a bulk material. (C) 2013 American Institute of Physics. [http://dx.doi.org/10.1063/1.4793657]
\end{abstract}

Perovskite manganites belong to the class of highly correlated electron systems, where phase separation controls a wide range of electronic and magnetic properties. ${ }^{1,2}$ A reduction of the size of these materials to the nanoscale leads to dramatic changes in their properties as the finite size, grain boundary, and surface strain effects can play an important role. ${ }^{3-8}$ The potential strength of strain effects was very recently evidenced in manganite thin films, as the Curie temperature was shown to be increased several times via orbital control resulting from appropriate strain. ${ }^{4}$ Also, some recent studies showed the influence of high strain fields in large volumes of CMR material $^{3,5-8}$ where surface strain in nanocrystalline grains substantially influences the magnetic phase formation. Exploring strain effects combined with composition distribution could establish a way to strongly tune the magnetic properties of bulk materials, beyond previous reports. ${ }^{8}$

In the present study, single phase nanocrystalline $\mathrm{La}_{0.95} \mathrm{Sr}_{0.05} \mathrm{MnO}_{3+\delta}$, which in bulk single-crystal form is a canted antiferromagnet, ${ }^{9,10}$ is here shown to present an unusual ferromagnetic transition at ordering temperature $290 \mathrm{~K}$ $\left(T_{C 1}\right)$ and a broad transition at $\sim 150 \mathrm{~K}\left(T_{C 2}\right)$. An analysis of electron energy loss spectroscopy (EELS) spectra and detailed high resolution transmission electron micrography (HRTEM) study confirm the oxygen homogeneity at the grain interface and the existence of strain in the system. The magnetic properties of the system are interpreted via the mean-field model, taking into account a distribution of $T_{C}$ with two main distinct transition temperatures, each with its

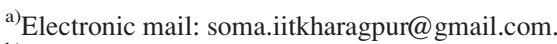

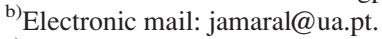

${ }^{c}$ Electronic mail: kalyanashis.de@gmail.com.
}

own degree of inhomogeneity. A combined effect of doping and the strain associated with is discussed to explain the tuning of properties of this bulk system.

Polycrystalline $\mathrm{La}_{0.95} \mathrm{Sr}_{0.05} \mathrm{MnO}_{3+\delta}$ samples were prepared via a chemical route, ${ }^{11}$ resulting in an average grain size of $\sim 120 \mathrm{~nm}$, obtained by scanning electron microscope. The single phase nature of the material was confirmed by powder X-ray diffraction, and the diffraction pattern is indexed by the orthorhombic structure (Pbnm) with $a=5.47(2) \AA, b=5.60(4) \AA$, and $c=7.71(1) \AA$. Fig. 1(a) shows HRTEM imaging of a typical grain, showing clear lattice resolved planes, implying good crystallinity inside the particle. The selected area electron diffraction (SAED) pattern, shown in the inset of Fig. 1(a), is consistent with the orthorhombic phase observed from X-ray diffraction. The majority of particles are thin and hexagonally shaped, with size in the range of $60-80 \mathrm{~nm}$, in a total size distribution ranging from 40 to $200 \mathrm{~nm}$. The general features of the observed grains are highly crystalline nature, with a varying relative proportion of $\mathrm{La}-$ and $\mathrm{Sr}$-ions, with $\mathrm{Sr}$ content up to 9 at. \% inside the grains. Bulk oxygen stoichiometry in the system was verified through iodometric titration. DC magnetization $(M)$ was measured in vibrating sample magnetometers (VSMs) with a field range from 0 to $10 \mathrm{~T}$.

Fig. 2 shows the temperature $(T)$ dependence of zero field cooled (ZFC) magnetization (MZFC) and field cooled (FC) magnetization (MFC) of the system at an applied magnetic field $(H)$ of $1 \mathrm{kOe}$. On decreasing temperature, a sharp increase of MZFC and MFC occurs below $290 \mathrm{~K}$, indicating that the system orders and attains a spontaneous magnetization at lower temperatures as discussed below. The lower inset of Fig. 2 shows the plot of temperature derivative of FC 

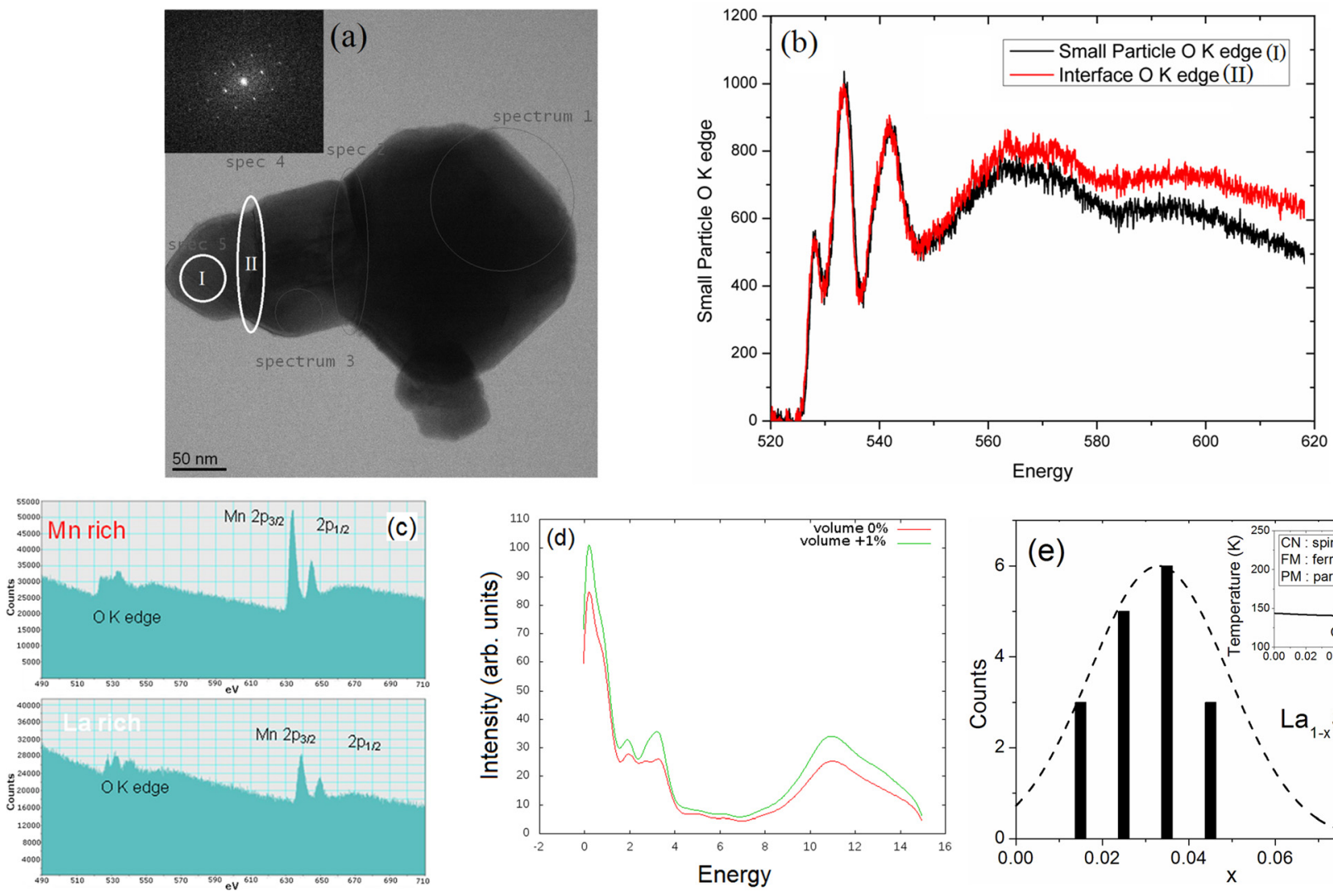

FIG. 1. (a) TEM image of grain, inset is the SAED. (b) Oxygen K edge spectra at intra grain (I) and interface (II), showing the homogeneity in oxygen across the grain boundary. (c) EELS of oxygen K edge and the white line ratio of the Mn L edge taken from the intra grain region. (d) Change in Mn L2,3 line inten sities with strain (corresponding to $1 \%$ volume change) obtained by (L)APW+lo density functional method (WIEN2k). (e) Histogram showing the variation of $\mathrm{Sr}$ content obtained by various EDX measurements, and fit to a Gaussian distribution. Inset shows the $\mathrm{La}_{1}{ }_{x} \mathrm{Sr}_{x} \mathrm{MnO}_{3}$ magnetic phase diagram for $x<0.1$ (adapted from Ref. 9).

magnetization $\left(d M_{F C} / d T\right)$ showing a local minimum at $T_{C 1} \sim 290 \mathrm{~K}$ indicated by the arrow. A rough preliminary analysis of the low-field behavior, by fitting the low-field magnetization data for $T \lesssim T_{C 1}$ by the critical behavior expression $M=B\left|T-T_{C 1}\right|^{\beta}$, where $\beta$ has the mean-field value of $1 / 2,{ }^{12}$ would suggest that about $40 \%$ of the magnetic ions are involved in the ordering with transition temperature near $T_{C 1}$, while the remaining ions present a combined AFM/ FM behavior, with a wide range of transition temperatures, around $T_{C 2}, \sim 150 \mathrm{~K}$. The absence of steepness in $d M_{F C} / d T$ (for $T<T_{C 1}$ ) denotes the onset of an inhomogeneous state in the system.

The plot of inverse susceptibility $(H / M)$ with temperature at the upper inset of Fig. 2 follows a linear behavior above $T_{C 1}$, in the measured range up to $315 \mathrm{~K}$, according to the Curie-Weiss law: $M / H=C /\left(T-\Theta_{W}\right)$, where $C$ and $\Theta_{W}$ are the Curie and Weiss constant, respectively. The obtained value of the effective paramagnetic moment $\left(\mu_{\text {eff }}\right)$ is $\sim 3.25 \mu_{B}$, with $\Theta_{W} \sim 290 \mathrm{~K}$, in good agreement with Curie temperature $\left(T_{C 1}\right)$. Considering the rigid Hund coupling within $\mathrm{Mn}^{3+}$ and $\mathrm{Mn}^{4+}$ in their high spin state and the 5 at. \% Sr doping, the theoretical effective paramagnetic moment $\left(\mu_{\text {eff }}\right)$ per formula unit can be estimated as $\sim 4.85 \mu_{B}$. The large difference between this and the experimentally obtained $\mu_{\text {eff }}$ may be due to oxygen nonstoichiometry as well as the surface disorder effect at the grains and/or magnetic clustering. A measure of nonstoichiometric oxygen by iodometric titration shows the excess oxygen to be $\sim 1.6 \%$ which, however, does not completely justify the experimentally observed value of $\mu_{\text {eff }}$. The low value of $\mu_{\text {eff }}$ can be justified if we take into account that the magnetization changes with temperature near $T_{C 1}$ will be mostly dependent on the Mn ions with $T_{C} \sim T_{C 1}$, establishing the values of $\Theta_{W}$ and $C$ of the Curie-Weiss law. Considering our previous estimation that $\sim 40 \%$ ions are ordering near $T_{C 1}$ and the $N$ (number of ions) parameter is changed adequately, the resulting corrected value of $\mu_{\text {eff }}$ is $4.76 \mu_{B}$, much closer to the expected value.

In order to further verify the surface disorder effect of the grains, we performed a detailed HRTEM study at different regions of the sample. We observe some regions which are highly homogeneous in elemental concentration and some inhomogeneous regions. In the inhomogeneous regions, typical HRTEM-EDX spectra show $\mathrm{Sr}$ content within $\sim 2-9$ at. $\%$ inside the grains and $\sim 1$ at. $\%$ at the interfaces. On the other hand, oxygen homogeneity is confirmed by EELS K-edge line at interface and intra-grain (Fig. 1(a)). This excludes the possibility of surface disorder effects to play a major role in the magnetic property of the system.

Detailed EELS showed (Fig. 1(b)) the difference in oxygen K-edge and white line ratio of $\mathrm{Mn}$ L-edge (ratio between the $2 p_{3 / 2}$ and $2 p_{1 / 2}$ lines) in Mn-rich and La-rich region in intra-grain space. These results point to the existence of strain in the structure at the atomic level, taking into account that the observed sharp and narrow XRD peaks, with sufficiently broad full width at half maxima to accommodate the 


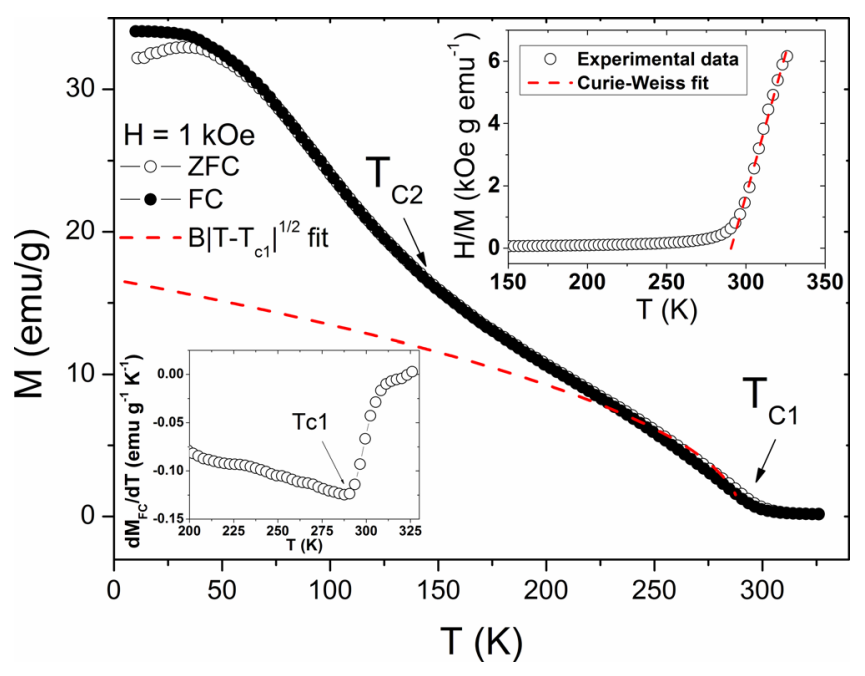

FIG. 2. The temperature dependence of ZFC and FC magnetization at $H \quad 1 \mathrm{kOe}$. Dashed line shows fit to $B\left|T \quad T_{C 1}\right|^{1 / 2}$. The lower inset shows $d M F C / d T$ dependence on $T$. The upper inset shows the plot of inverse sus ceptibility $H / M$ as a function of temperature and fit to the Curie Weiss law.

compositional range of interest, to establish a single phase structure. In order to quantitatively interpret a dependence of EELS spectra on strain effects, we have carried out first principle calculations of the undoped parent $\mathrm{LaMnO}_{3}$ system using the (L)APW+lo density functional method implemented in WIEN2k, using the LDA, with no spinpolarization and with the initial-state approximation. ${ }^{13}$ This gives us a clear change in the intensity as well as the shape of the peaks of Mn L2,3 when a volume change of $1 \%$ is applied on the lattice as shown in Fig. 1(d). The use of $a b$ initio simulations has been shown to adequately predict strain effects in energy loss spectra (see Ref. 14 for a detailed use of this approach). We can therefore conclude that there are strong localized strain regions within the sample, which can promote an increase in $T_{C}$ values as was recently observed in thin film nanostructures of similar compounds. ${ }^{4}$ First principle calculations of superlattice structures of $\mathrm{La}_{2 / 3} \mathrm{Sr}_{1 / 3} \mathrm{MnO}_{3}$ (LSMO) and $\mathrm{BaTiO}_{3}$ (BTO) layers, which possess a weak density of states at Fermi level in the dominant LSMO spin orientation, have predicted strain dependent ferromagnetic phases and also a high Curie temperature, compared to bulk samples with no octahedral tilt. Experimental results of laser molecular beam epitaxy (MBE) deposited samples confirmed the emergence of regions with higher $T_{C}$, much above the bulk values. In the present system, a global random observation (of $\sim 20$ observations done at different parts of the sample and in different sets to have an overall behavior of the sample in larger scale) in the non-homogeneous regions inside the grains of the sample shows the $\mathrm{Sr}$ content variation as given in Fig. 1(e). This $\mathrm{Sr}$ inhomogeneity in the intra-grain regions changes the behavior from metallic to insulating. These regions may therefore be subject to the appropriate strain, associated with this doping level, controlling the orbital ordering. This can favor the extended ferromagnetism and therefore result in a high magnetic ordering temperature of $290 \mathrm{~K}$, as compared to the expected canted antiferromagnetic behavior of single crystal samples, with $T_{N} \sim 150 \mathrm{~K} .{ }^{9}$ As seen in Fig. 1(e), there is a higher possibility of $\mathrm{Sr}$ to be in a low concentration state although the average value is close to the stoichiometric proportion. The system also preserves some regions with homogeneous stoichiometric elemental concentration. This elemental inhomogeneity in the system also explains the distribution of $T_{C}$ in the range $T \sim T_{C 2}$ as shown in the inset of Fig. 1(e). A change of $T_{C}$ from minimum to maximum can be obtained with this $\mathrm{Sr}$ distribution $\left(\int_{T_{C \text { min }}}^{T_{C}} f\left(T_{C}\right) d T_{C}\right.$ $\left.=\int_{x \text { min }}^{x} g(x) d x\right)$, and the major change comes in the intermediate region, which, we believe, is under the appropriate strain to preserve this magnetic ordering. This elemental inhomogeneity in the system also accounts for the presence of magnetic frustration that can give rise to a glassymagnetic state in the system as we have obtained at low temperature but is not illustrated here (a typical signature however is seen in Fig. 2 towards low temperature).

As shown in Fig. 2, the overall $M$ vs $T$ behavior cannot be described by a single broad $T_{C}$ distribution, as there are two clear regions where $M$ decreases, near $T_{C 1}$ and $T_{C 2}$. In the light of previous work, where the effects of inhomogeneity in the magnetic properties of manganites were adequately reproduced using $T_{C}$ distributions and a meanfield approach, ${ }^{15}$ simulations of an inhomogenous mean-field system with spin 2 were performed, in order to have insight on the ordering temperature distribution that would reproduce the observed full $M(H, T)$ behavior. Two ordering temperature regions were considered, each with its own width, throughout the experimental $(H, T)$ range. As an example, $M$ vs $T$ behavior at $10 \mathrm{kOe}$ (Fig. 3(a)) was adequately reproduced with a high-temperature $T_{C 1} \sim 300 \mathrm{~K}$ (100 K FWHM) and $T_{C 2} \sim 150 \mathrm{~K}(140 \mathrm{~K}$ FWHM), as shown in Fig. 3(b). The combined Gaussian distributions of these two temperatures used in the simulation are directly related
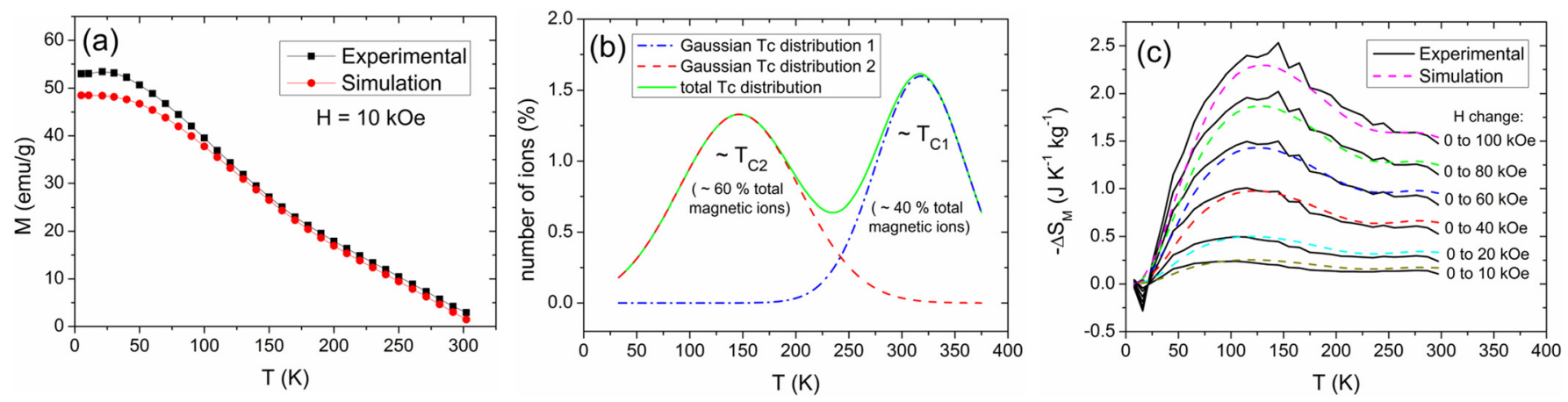

FIG. 3. (a) $M$ vs. $T$ behavior of $\mathrm{La}_{0.95} \mathrm{Sr}_{0.05} \mathrm{MnO}_{3}$ system at $H \quad 10 \mathrm{kOe}$, from experiment and simulation. (b) Distribution of $T_{C}$ 's used in the mean field $M(H, T)$ simulations. (c) Magnetocaloric effect (entropy change) of the same system from experimental data and simulation. 
to the $\mathrm{Sr}$ distribution of this system that influences the magnetic behavior. It is found that around $40 \%$ of magnetic ions are in the high $T_{C}$ state, which is in good agreement with the low-field magnetization data analysis of Fig. 2, confirming that the majority of the system has a low Sr concentration contributing higher magnetization value at $T_{C 2}$ while $\sim 40 \%$ of the system has higher ordering temperature (high $\mathrm{Sr}$ content). This is again confirmed from our EDX study in HRTEM that shows the higher probability of Sr near 3 at. \% (Fig. 1(e)). In the low temperature region in Fig. 3(a), well below the $T_{C}$ distribution range, the simulation deviates somewhat from the experimental results as it considers the system as ferromagnetic only and therefore does not take into account the competing magnetic interactions. This is reflected as lowering of magnetization from the experimental behavior. To better interpret the full $M(H, T)$ dependence of the system, the magnetic entropy change $\left(\Delta S_{M}(H, T)\right)$ of the system, obtained experimentally, is compared to the simulated mean-field system, as shown in Fig. 3(c).

An analysis of $\Delta S_{M}$ is here convenient as magnetic ordering transitions are clearly seen, together with the $H$ dependence, throughout the acquired magnetic data, from 20 to $300 \mathrm{~K}$ and from 0 to $100 \mathrm{kOe}$. The usefulness of $\Delta S_{M}$ as a global consistency verification at a transition results mostly from the fact that $\Delta S_{M}$ peaks at $T_{C}$ in the whole field range, in contrast to a simple $d M / d T$ analysis at fixed field, in which peaks strongly shift to higher $T$ values with applied magnetic field. The known dependence of $\Delta S_{M}$ on $T_{C}{ }^{2 / 3}$ (Ref. 16) confirms that the distribution should have a higher maximum at $T_{C 1}$, in order to result in $\Delta S_{M}$ peaks of similar intensity around $T_{C 1}$ and $T_{C 2}$, which is verified in the data of Fig. 3(c). Fig. 3(c) also shows that the experimental $\Delta S_{M}$ at temperatures below $\sim 30 \mathrm{~K}$ becomes negative and is therefore different from simulation. This provides further evidence for the influence of glassy behavior at low temperatures, which are not taken into account in the simulation. The magnetization and magnetic entropy simulations therefore confirm the dynamics of the system and its predominant ferromagnetic behavior from $\sim 50 \mathrm{~K}$ to $300 \mathrm{~K}$ following the two main ordering temperatures at $T_{C 1}$ and $T_{C 2}$.

In conclusion, we have shown that it is possible to strongly tune the magnetic properties of bulk materials, from the combined effects of localized strain and local scale inhomogeneity. We have studied the low-Sr doped composition $\mathrm{La}_{0.95} \mathrm{Sr}_{0.05} \mathrm{MnO}_{3+\delta}$ (a canted antiferromagnet, according to previous studies ${ }^{9}$ ) and have shown it to be tunable to be ferromagnetic with a $T_{C}$ near room temperature. This strong tuning of magnetic behavior opens new possibilities of optimizing the magnetic properties of bulk materials as was previously shown to be possible in thin film LSMO-BTO strained nanostructures. ${ }^{4}$

The authors acknowledge the financial support from FCT through Grant No. SFRH/BPD/39262/2007 (S. Das), SFRH/BPD/63942/2009 (J. S. Amaral), UGC-Dr. D. S. Kothari Post-Doctoral Fellowship under the Project No. F.42/2006(BSR)/13-307/2008(BSR) (K. De) and from FCT through Grant Numbers SFRH/BPD/47128/2008 (K. De) and SFRH/BD/63698/2009 (A. Roy), and partial funding from the program COMPETE-FEDER under project PTDC/ FIS/105416/2008.

${ }^{1}$ E. Dagotto, T. Hotta, and A. Moreo, Phys. Rep. 344, 1 (2001).

${ }^{2}$ K. H. Ahn, T. Lookman, and A. R. Bishop, Nature (London) 428, 401 (2004).

${ }^{3}$ C. Dhital, C. de la Cruz, C. Opeil, A. Treat, K. F. Wang, J. Liu, Z. F. Ren, and S. D. Wilson, Phys. Rev. B 84, 144401 (2011).

${ }^{4}$ A. Sadoc, B. Mercey, C. Simon, D. Grebille, W. Prellier, and M. Lepetit, Phys. Rev. Lett. 104, 046804 (2010).

${ }^{5}$ R. Mahesh, R. Mahendiran, A. K. Raychaudhuri, and C. N. R. Rao, Appl. Phys. Lett. 68, 2291 (1996).

${ }^{6}$ E. Rozenburg, S. Banerjee, I. Felner, E. Sominski, and A. Gedanken, J. Non Cryst. Solids 353, 817 (2007).

${ }^{7}$ M. V. Kharlamovaa and A. Arulraj, JETP Lett. 89, 301 (2009).

${ }^{8}$ K. S. Shankara, S. Kara, G. Subbannab, and A. Raychaudhuri, Solid State Commun. 129, 479 (2004).

${ }^{9}$ A. Urushibara, Y. Moritomo, T. Arima, A. Asamitsu, G. Kido, and Y. Tokura, Phys. Rev. B 51, 14103 (1995).

${ }^{10} \mathrm{~J}$. B. Goodenough, in Handbook on the Physics and Chemistry of Rare Earths, edited by K. A. Gschneidner, Jr., J. C. G. Bunzli, and V. K. Pecharsky (Elsevier, 2003), Chap. 214, Vol. 33.

${ }^{11}$ K. De, R. Ray, R. N. Panda, S. Giri, H. Nakamura, and T. Kohara, J. Magn. Magn. Mater. 288, 339 (2005).

${ }^{12}$ H. E. Stanley, Introduction to Phase Transitions and Critical Phenomena (Clarendon, Oxford, 1971).

${ }^{13}$ P. Blaha, K. Schwarz, G. Madsen, D. Kvasnicka, and J. Luitz, WIEN2k, An Augmented Plane Wave Plus Local Orbitals Program for Calculating Crystal Properties, Technische Universitat Wien, Vienna, 2001. See http://www.wien2k.at/.

${ }^{14}$ V. J. Kesat, M. J. Kappers, and C. J. Humphreys, J. Microsc. 210, 89 (2003).

${ }^{15}$ J. S. Amaral, P. B. Tavares, M. S. Reis, J. P. Araújo, T. M. Mendonça, V. S. Amaral, and J. M. Vieira, J. Non Cryst. Solids 354, 5301 (2008).

${ }^{16}$ J. H. Belo, J. S. Amaral, A. M. Pereira, V. S. Amaral, and J. P. Araújo, Appl. Phys. Lett. 100, 242407 (2012). 